\title{
TRIM31 promotes proliferation, invasion and migration of glioma cells through Akt signaling pathway
}

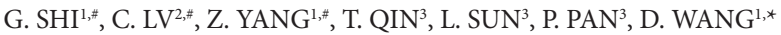 \\ ${ }^{1}$ Department of Pathology, Medical College, Nantong University, Nantong, China; ${ }^{2}$ Department of Physical Education, Nantong University, \\ Nantong, China; ${ }^{3}$ Affiliated Hospital of Nantong University, Nantong University, Nantong, China \\ ${ }^{*}$ Correspondence: donglinwang@126.com \\ "Contributed equally to this work.
}

Received January 6, 2019 / Accepted March 20, 2019

\begin{abstract}
This study is intended to investigate the role of Tripartite Motif (TRIM) 31 in glioma. Immunohistochemistry and western blot analysis showed that TRIM31 was overexpressed in high-grade glioma tissues. Univariate survival analysis indicated that high expression of TRIM31 was related to short survival time of glioma patients. Multivariate survival analysis demonstrated that TRIM31 was an independent prognostic factor for glioma patients. In addition, through the experiments on glioma cell lines, we found that after silencing or overexpressing TRIM31 expression, the proliferation, invasion and migration of glioma cells could be downregulated or upregulated through Akt signaling pathway. In short, our study suggests that TRIM31 may be an effective target for glioma intervention.
\end{abstract}

Key words: glioma, TRIM31, prognosis, Akt

Glioma is one of the most malignant primary tumors of the central nervous system [1], including astrocytoma, oligodendroglioma, ependymoma, anaplastic astrocytoma, glioblastoma and so on [2]. The biological feature of glioma cells is its strong ability to proliferate, migrate and invade normal adjacent tissues [3]. Despite the comprehensive application of surgery, radiotherapy and chemotherapy, the prognosis of glioma patients is still not optimistic [4]. Highgrade glioma patients are prone to recurrence and metastasis, with the median survival time of only about 15 months [5]. In the latest classification of glioma in 2016, more emphases were placed on the characteristics of chromosomes and genes changes, which made the treatment more individualized and specific [6]. In addition, recent researches in the past decade suggested that finding precise molecular markers and better understanding of the biological behaviors of glioma cells were of a great benefit to the treatment of patients, rather than limited understanding of tumor morphology and histology $[7,8]$.

There are more than 70 members of TRIM family, which have been reported to contribute a lot to cell proliferation, differentiation and apoptosis, regulate transcription of other genes, and participate in proteins ubiquitination [9]. The common structure characteristics of TRIM family are a conserved RING finger, B-box and coiled-coil domains [10]. TRIM31, a member of TRIM family, serves as an E3 ubiquitin-protein ligase, which was originally identified as a gene induced by growth-suppressive retinoid [11]. It has been reported that TRIM31 plays a role in tumor progress, such as promoting the growth of colorectal cancer [12], gallbladder cancer [13] and hepatocellular carcinoma [14], while suppressing the carcinogenesis of non-small cell lung cancer [15]. However, the molecular mechanism of TRIM31 in the development of glioma remains unknown.

In this study, we explored the expression of TRIM31 in glioma tissues and evaluated its significance on glioma patients' prognosis. Besides, the molecular mechanism and biological functions of TRIM31 was performed on glioma cells lines in vitro, through which we discovered that TRIM31 affected proliferation, invasion and migration of glioma cells via Akt signaling pathway.

\section{Materials and methods}

Patients and tissue samples. Obtained from Affiliated Hospital of Nantong University from 2011-2013, the glioma specimens were collected from newly diagnosed patients, without treatments of radiotherapy or chemotherapy before 
operation. All the 80 samples we used in our experiments were from astrocytoma patients, which had been approved by Human Research Ethics Committee. Specimens for immunohistochemistry analysis were fixed in 10\% formalin and embedded in paraffin, while tissues for western blot analysis were frozen at $-80^{\circ} \mathrm{C}$ immediately after surgery.

Antibodies. Anti-TRIM31 (ab244481 applicated to immunohistochemistry, ab98207 applicated to western blot), anti-glyceraldehyde-3-phosphate dehydrogenase (GAPDH, ab9484) and anti-proliferating cell nuclear antigen (PCNA, ab18197) antibody were from Abcam; anti-Ki-67 (sc-23900) and anti-E-cadherin antibody (sc-8426) were purchased from Santa Cruz Biotechnology; anti-Akt antibody (C67E7), anti-HA (C29F4), p-Akt(S473) (D9E), p-Akt(T308) (D25E6) were from Cell Signaling Technologies.

Immunohistochemistry. We used biotin amplification method. In short, after 6 hours of continuous baking, paraffin sections were dewaxed. We used the pressure cooker antigen repair method to retrieve the antigen. After soaking in $0.3 \%$ hydrogen peroxidase to block endogenous peroxides, the sections were incubated with anti-TRIM31 antibody overnight at $4{ }^{\circ} \mathrm{C}$. No immune serum was used as negative control, while Ki-67 was used as positive control. The next day, after washed by phosphate buffer saline (PBS), the sections were incubated with the second antibodies according to the instructions. At last, they were 3,3-diaminobenzidine (DAB) and hematoxylin stained and mounted in resin mount after dehydrated.

We invited two pathologists to evaluate the immunostaining independently in a blinded manner, who didn't have any previous information about the specimens. Intensity was estimated as follows: 0 (negative staining); 1 (weak staining); 2 (moderate staining); 3 (strong staining). Percentage of tumor cells stained positive was scored as follows: $0(<10 \%)$; 1 (11-25\%); 2 (26-50\%); 3 (51-75\%); 4 (>75\%). Then the two scores were added, and $0-3$ were counted as low expression, while 4-7 were counted as high expression.

Western blot analysis. Proteins were mixed with $2 \mathrm{x}$ loading buffer to be the loading samples, which were electrophoresed by $10 \%$ sodium dodecyl sulfate-polyacrylamide gel electrophoresis (SDS-PAGE) and then transferred to polyvinylidene fluoride (PVDF) membranes. After blocked in 5\% nonfat milk, the membranes were incubated with primary antibodies overnight at $4{ }^{\circ} \mathrm{C}$. The next day, after washed with PBS, they were incubated with secondary antibodies at room temperature for 2 hours. At last, they were detected by an enhanced chemiluminescence (ECL) detection system.

Cell culture. Human glioblastoma cell lines H4, A172, U87MG and U251MG were purchased from cell library of China Academy of Science, which were grown in Dulbecco's Modified Eagle Medium (DMEM) with 10\% fetal bovine serum (FBS) at $37^{\circ} \mathrm{C}$ with $5 \% \mathrm{CO}_{2}$. The cell medium was changed every $2-3$ days due to the cells living conditions.

Immunocytofluorescence. Firstly, cells were seeded on coverslips in a 24 -well plate overnight. After washed with PBS, cells were fixed with $4 \%$ paraformaldehyde for $30 \mathrm{~min}$ and blocked in 5\% bovine serum albumin (BSA) for $40 \mathrm{~min}$. Then, cells were incubated with anti-TRIM31 antibody overnight. The next day, after washed with PBS, cells were incubated with secondary antibodies for 2 hours at room temperature. For DNA staining, cells were incubated with Hoechst 33342 dye. At last, coverslips were put face down on slides to be detected by a Leica fluorescence microscope.

Expression plasmid and transient transfection. Over expression plasmid of TRIM31 was constructed by cloning the PCR-amplified full-length human TRIM31 cDNA into the pMSCV retrovirus. Short hairpin RNA (ShRNA) of TRIM31 oligonucleotides sequences were synthesized by Shanghai Genechem (China). The sequences were as follows: shTRIM31-1, UUCCCGUCAAAGGAAGUUUGG; shTRIM31-2, UAUGAUGGACUCAUGCCUUGC; ShTRIM31-3, UUAGAAGUUACGUGCAGUCUG. The sequence of control shRNA was 5'-CCCCUUUUAAAAGGGGCCCGG-3'. Cell Transient transfection experiments were performed with Lipofectamine 2000 .

Cell proliferation assay. After seeded in 96-well cell culture cluster plates, cells were processed by a commercial Cell Counting Kit (CCK)-8 (Dojindo, Kumamoto, Japan) with the manufacturer's instructions. Then, they were measured the absorbance at the wavelength of $490 \mathrm{~nm}$ by ImmunoMini NJ-2300.

Invasion assay. We used a transwell chamber (Corning, 8 - $\mu \mathrm{m}$ pore size) to perform the cell invasion experiment. Cells with a proper density were cultured in DMEM without FBS in the upper chamber, which has a polycarbonate membrane. DMEM with $10 \%$ FBS was put in the lower chamber. After cultured overnight, top (non-migrated) cells were removed and bottom (migrated) cells were fixed, stained with crystal violet and counted immediately.

Wound-healing assay. Cells were seeded in 6-well plates with different processing methods. 48 hours incubation after a line was scratched within the confluent cell layer with a 10 $\mu \mathrm{l}$ micropipette tip, images were taken by an inverted Leica phase-contrast microscope.

Statistical analysis. All statistical analyses were performed using SPSS 13.0 software. Kaplan-Meier (K-M) curves and log-rank test were used for univariate survival analysis. Cox's proportional hazards regression model was used for multivariate survival analysis. The Chi square $\left(\chi^{2}\right)$ test was used to analyze the relationship between TRIM31 expression and the clinicopathological features. Spearman rank correlation analysis was used to evaluate the immunohistochemistry results of TRIM31 and Ki-67. The significance of differences in multiple groups was analyzed by one-way ANOVA. Data were presented as mean \pm standard deviations (SD). All the experiments were carried out at least three times independently. P-values $<0.05$ were considered to be statistically significant. 


\section{Results}

Relationship between TRIM31 expression with glioma grade, and TRIM31 significance in glioma patients' survival analysis. We evaluated TRIM31 expression in seven fresh glioma tissues from Grade II to Grade IV by western blot analysis, which could be used to get the expression information of specific antigens [16]. It's revealed in Figure 1A that TRIM31 expression was significantly higher in high grade glioma tissues. Besides, immunohistochemistry of TRIM31 expression in 80 glioma samples further confirmed that TRIM31 was overexpressed in high grade gliomas (Figure 1B), since immunohistochemistry can be used to localize, characterize and quantify antigens in tissues and cells [17]. From the Spearman rank test result in Figure $1 \mathrm{C}$, it's obvious that there was a positive relationship between TRIM31 and Ki-67 expression ( $\mathrm{r}=0.779, \mathrm{p}<0.001)$, and Ki-67 is widely used as an ancillary tool for the diagnosis and prognosis of glioma [18]. The clinicopathological features of the 80 samples were presented in Table 1, which included 25 cases of Grade II gliomas, 31 cases of Grade III gliomas and 24 cases of Grade IV gliomas. $\chi^{2}$ test results showed that TRIM31 expression differed between different grades of gliomas $\left(\chi^{2}=27.225, \mathrm{p}<0.001\right)$. K-M survival curves and log-rank test results indicated that higher expression of TRIM31 was associated with a poorer prognosis of glioma

A

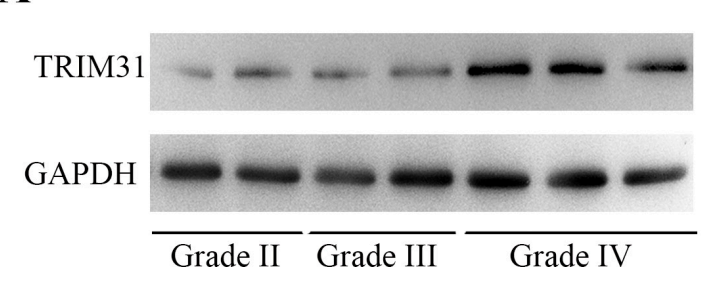

B

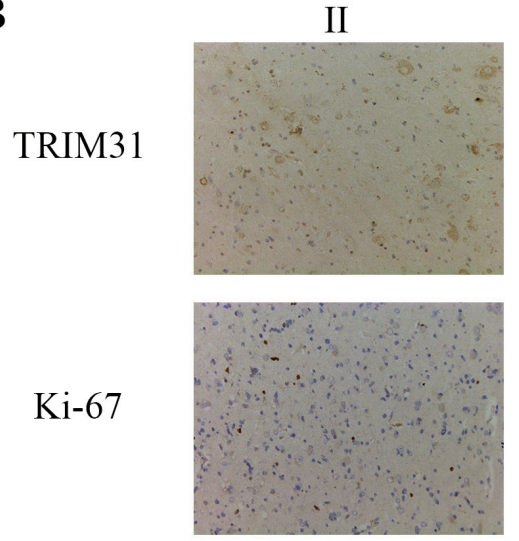

C

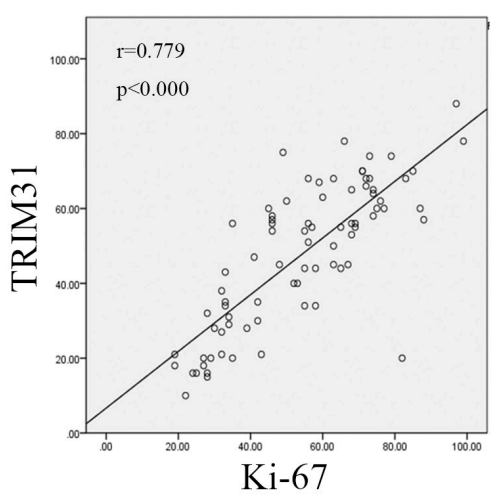

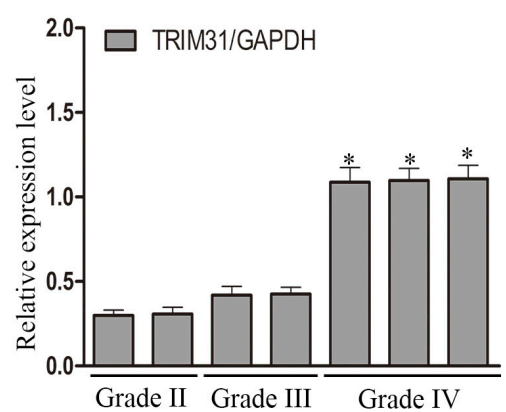

III
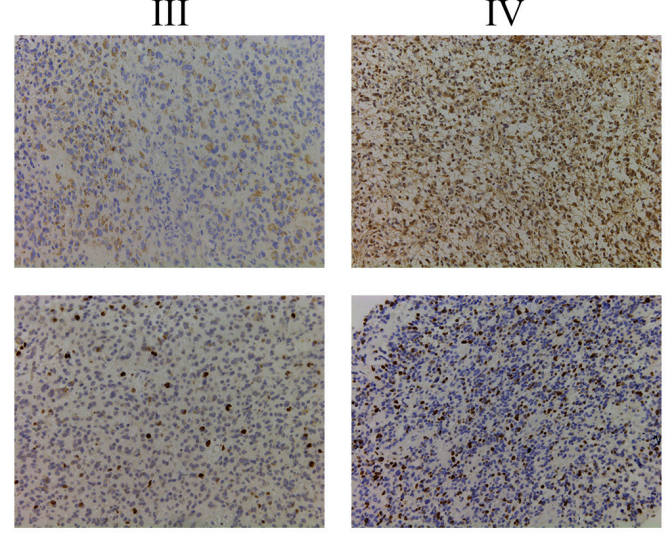

D

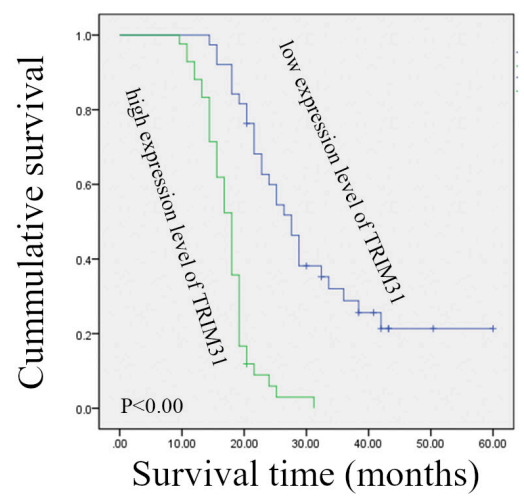

Figure 1. TRIM31 expression in glioma and its significance on glioma patients' survival. A) Western blot results of TRIM31 in seven fresh glioma tissues. Bar charts were TRIM31 compared to GAPDH. ${ }^{\star} \mathrm{p}<0.05$, compared to Grade II. B) Immunohistochemistry results of TRIM31 and Ki-67. C) Scatter plots indicated TRIM31 and Ki-67 expression in immunohistochemistry. (D) K-M survival curve for high and low TRIM31 expression in 80 glioma patients. Patients with high expression of TRIM31 had a poorer survival, $\mathrm{p}<0.00$. 
Table 1. TRIM31 expression and clinical pathology characteristics in 80 cases of glioma specimens.

\begin{tabular}{|c|c|c|c|c|c|}
\hline \multirow{2}{*}{ Variable } & \multirow{2}{*}{ Total } & \multicolumn{2}{|c|}{ TRIM31 expression } & \multirow{2}{*}{$\chi^{2}$ value } & \multirow{2}{*}{ p-value } \\
\hline & & Low & High & & \\
\hline \multicolumn{6}{|l|}{ Age (years) } \\
\hline$<40$ & 36 & 16 & 20 & 0.486 & 0.486 \\
\hline$\geq 40$ & 44 & 23 & 21 & & \\
\hline \multicolumn{6}{|l|}{ Gender } \\
\hline Female & 47 & 24 & 23 & 0.244 & 0.621 \\
\hline Male & 33 & 15 & 18 & & \\
\hline \multicolumn{6}{|l|}{ Tumor location } \\
\hline Frontal & 17 & 8 & 9 & 0.320 & 0.956 \\
\hline Parietal & 39 & 20 & 19 & & \\
\hline Occipital & 14 & 6 & 8 & & \\
\hline Temporal & 10 & 5 & 5 & & \\
\hline \multicolumn{6}{|l|}{ Tumor size $(\mathrm{cm})$} \\
\hline$<4$ & 22 & 11 & 11 & 0.019 & 0.890 \\
\hline$\geq 4$ & 58 & 28 & 30 & & \\
\hline \multicolumn{6}{|l|}{ WHO Grade } \\
\hline II & 25 & 23 & 2 & 27.225 & $0.000^{*}$ \\
\hline III & 31 & 9 & 22 & & \\
\hline IV & 24 & 7 & 17 & & \\
\hline \multicolumn{6}{|l|}{ Extent of resection } \\
\hline Biopsy & 39 & 17 & 22 & 0.811 & 0.667 \\
\hline Total resection & 13 & 7 & 6 & & \\
\hline Subtotal resection & 28 & 15 & 13 & & \\
\hline
\end{tabular}

Pearson $\chi^{2}$ test for statistical analysis. ${ }^{*} \mathrm{p}<0.05$
Table 2. Contribution of various potential prognostic factors to survival by Cox regression analysis on 80 glioma specimens.

\begin{tabular}{lccc}
\hline Characteristic & Hazard ratio & $\mathbf{9 5 \%}$ CI & p-value \\
\hline Age & 1.231 & $0.724-2.094$ & 0.442 \\
Gender & 1.365 & $0.792-2.353$ & 0.263 \\
Tumor location & 0.945 & $0.687-1.299$ & 0.727 \\
Tumor size & 1.373 & $0.719-2.622$ & 0.336 \\
WHO Grade & 4.740 & $2.882-7.796$ & $0.000^{*}$ \\
Extent of resection & 0.987 & $0.735-1.325$ & 0.929 \\
TRIM31 expression & 2.845 & $1.585-5.108$ & $0.000^{*}$ \\
\hline
\end{tabular}

CI: confidence interval. ${ }^{*} \mathrm{p}<0.05$

patients $(\mathrm{p}<0.001)$ (Figure 1D). From the results of multivariate survival analysis in Table 2, we found that TRIM31 was an independent prognostic factor of glioma $(\mathrm{p}<0.001)$.

TRIM31 expression and location in glioma cells. From the western blot results in Figure 2A, it's clear that TRIM31 expression was higher in U87 MG and U251 MG cells, while its expression was lower in $\mathrm{H} 4$ and A172 cells. Cellular immunofluorescence is widely used for the localization of specific antigens [19], the results of which showed that TRIM31 was mainly expressed in cytoplasm of glioma cells (Figure 2B).

Silencing TRIM31 expression and its effects on other proteins. We transfected control shRNA and shRNAs of TRIM31 to U87 MG cells, which has a high expression of

$\mathbf{A}$
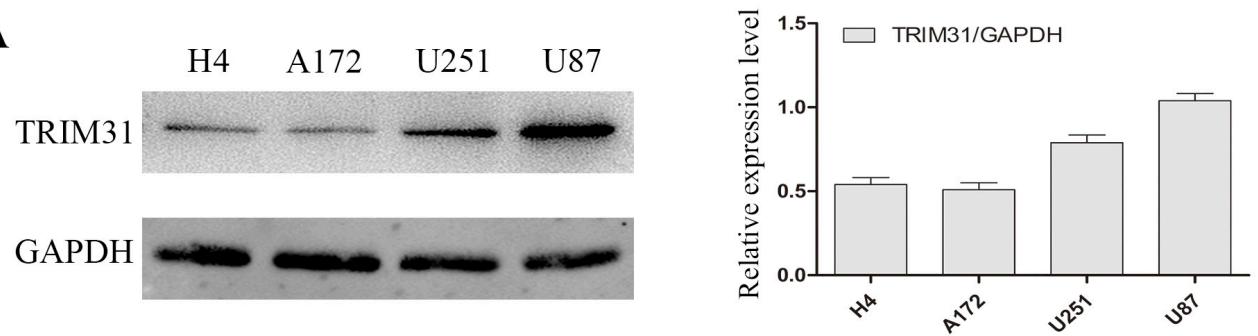

B

DAPI

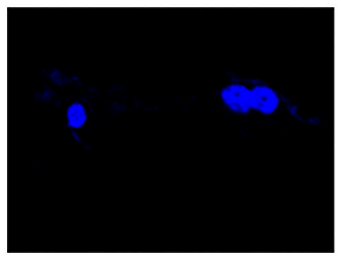

U87

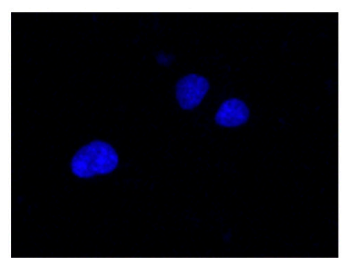

TRIM31
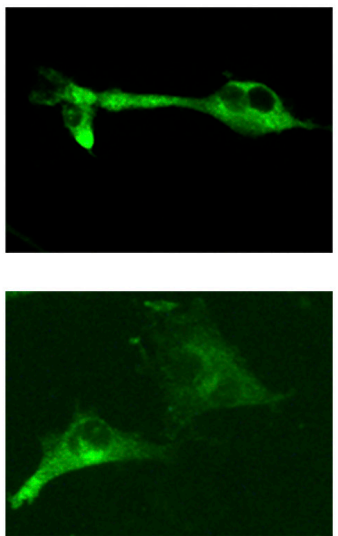
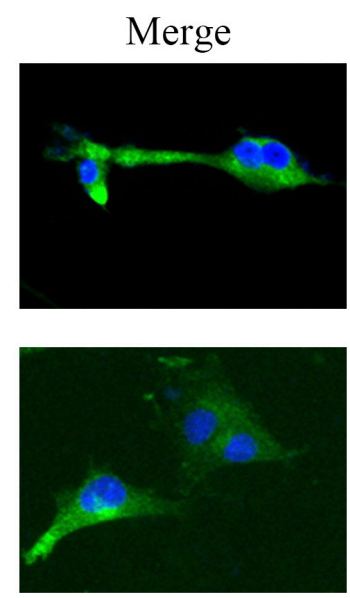

Figure 2. TRIM31 expression and location in glioma cells. A) Western blot results of TRIM31 in four glioma cells lines. Bar charts were TRIM31 compared to GAPDH. B) Immunocytofluorescence results of TRIM31 in U87 MG and H4 cells. 


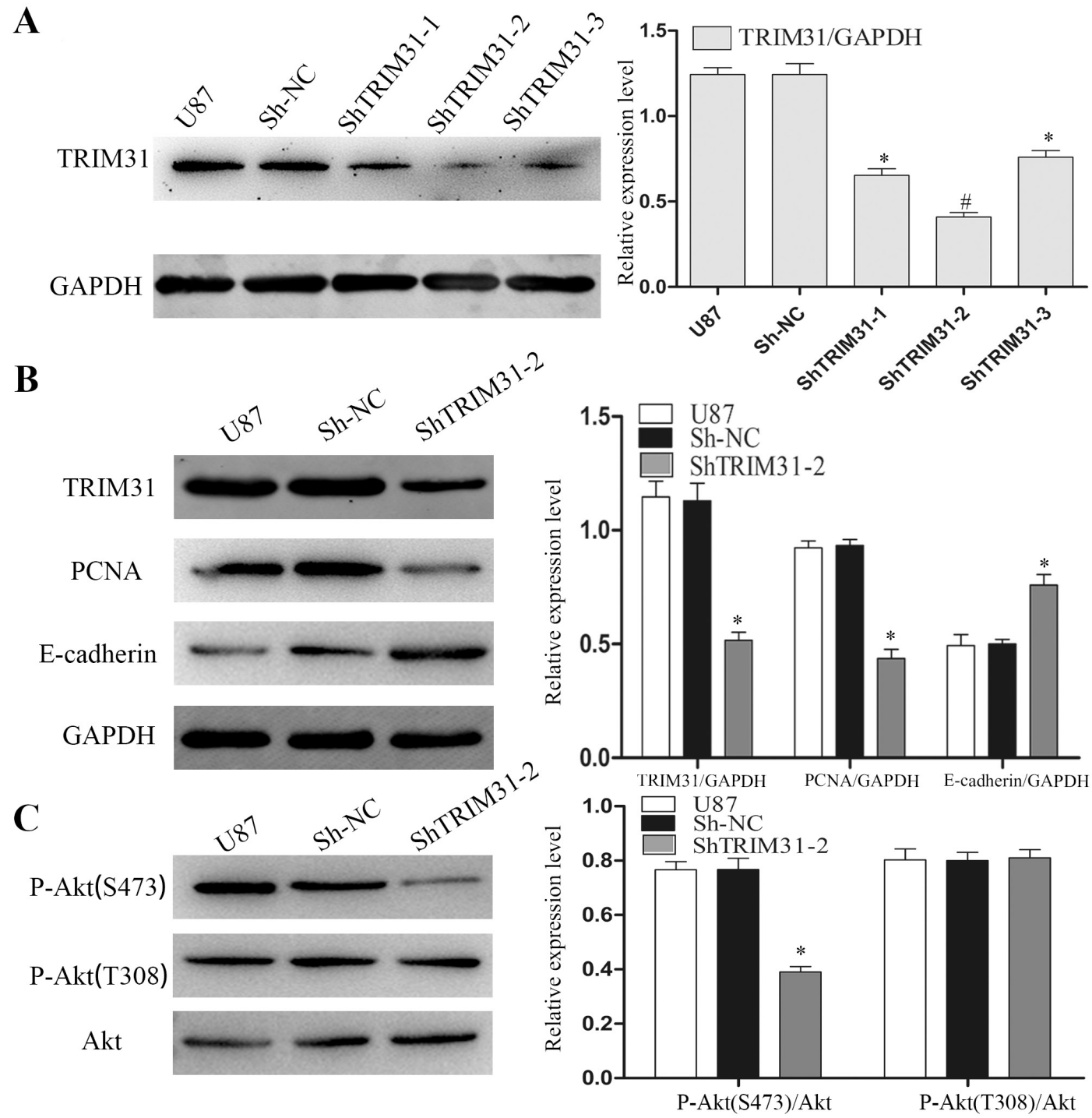

Figure 3. Knockdown of TRIM31 and its effects on other proteins. A) Knockdown efficiency of different shRNAs of TRIM31. Bar charts were TRIM31 compared to GAPDH. ${ }^{\star} \mathbf{p}<\mathbf{0 . 0 5}, \# p<0.01$, compared to sh-NC. B) Effects of silencing TRIM31 on PCNA and E-cadherin expression. Bar charts were TRIM31, PCNA and E-cadherin compared to GAPDH. ${ }^{\star} \mathrm{p}<0.05$, compared to sh-NC. C) Effects of silencing TRIM31 on Akt signaling pathway. Bar charts were P-Akt(S473) and P-Akt(T308) compared to Akt. ${ }^{*} \mathrm{p}<0.05$, compared to sh-NC.

TRIM31. From the results of Figure 3A, it is apparent that shTRIM31-2 showed best knockdown efficiency. Next, we examined expression of PCNA, E-cadherin by western blot between groups of U87, sh-NC and shTRIM31-2. Compared to the group of sh-NC, PCNA expression was decreased while E-cadherin was increased in the group of shTRIM31-2 (Figure 3B). PCNA was an important marker of cell proliferation, and E-cadherin was a major indicator of migration and invasion. Besides, we also examined the effect of downregulation of TRIM31 on Akt signaling pathway. It's revealed that P-Akt(S473) was downregulated after silencing TRIM31 expression, while no effect was observed in P-Akt(T308) (Figure 3C).
Knockdown of TRIM31 inhibited proliferation, invasion and migration of glioma cells through Akt signaling pathway. After transfection with shTRIM31, U87 MG cells were dealt with Akt agonist IGF-1. The groups of cells in different processing ways were shown in Figure 4A, from the western blot results in which we could see that downregulation of PCNA and P-Akt(S473) and upregulation of E-cadherin after transfection of shTRIM31 were reversed after both procession of shTRIM31 and IGF-1. However, downregulation of TRIM31 was not affected by IGF-1. We next used CCK-8 analysis, transwell invasion assay and wound healing assay, which were commonly applied to analyze cells proliferation, invasion and migra- 


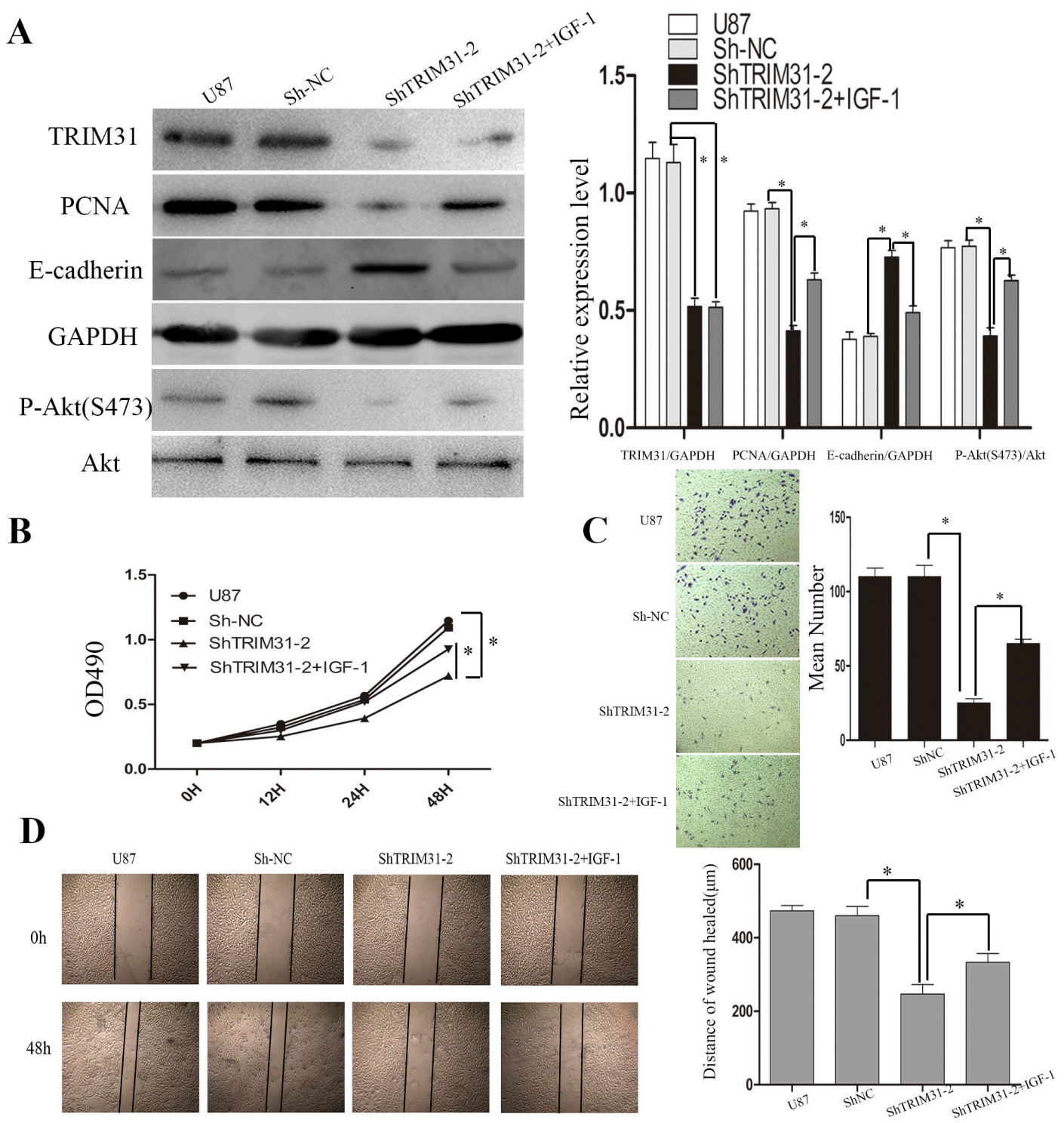

Figure 4. Effects of silencing TRIM31 and Akt agonist IGF-1 on proliferation, invasion and migration of glioma cells. A) Western blot results of different procession of U87 MG cells. Bar charts were TRIM31, PCNA, E-cadherin compared to GAPDH and P-Akt (S473) compared to Akt. ${ }^{*} \mathrm{p}<0.05$. B) CCK-8 analysis of different procession of U87 MG cells. ${ }^{\star}$ p $<0.05$. C) Transwell invasion assay of different procession of U87 MG cells. Bar charts were quantization results of transwell invasion assay. ${ }^{*} \mathbf{p}<0.05$. D) Wound-healing assay of different procession of U87 MG cells. Bar charts were quantization results of wound-healing assay. ${ }^{*} \mathrm{p}<0.05$.

tion [20]. It's revealed that inhibition of proliferation, invasion and migration of glioma cells induced by knockdown of TRIM31 could be abated by Akt agonist IGF-1 (Figures 4B-D).

Upregulation of TRIM31 promoted proliferation, invasion and migration of glioma cells through Akt signaling pathway. We transfected the HA labeled TRIM31 overexpression plasmid into $\mathrm{H} 4$ glioma cells, which has a low expression of TRIM31 (Figure 5A). We also treated H4 cells transfected with TRIM31 overexpression plasmid with Akt antagonist LY294002. The groups of cells in different processing ways were shown in Figure 5B, western blot analysis in which showed that upregulation of PCNA and P-Akt(S473) and downregulation of E-cadherin caused by upregulation of TRIM31 were reversed after both procession of TRIM31 overexpression and LY294002. However, upregulation of TRIM31 was not affected by LY294002. It is exhibited by CCK- 8 analysis, transwell invasion assay and wound healing assay that promotion of proliferation, invasion and migration of glioma cells induced by upregulation of TRIM31 could be weakened by Akt antagonist LY294002 (Figures 5C-E). 


\section{Discussion}

Glioblastoma multiforme (GBM), as the Grade IV glioma, is one of the most intractable and challenging cancers despite application of multimodality treatments, including maximal safe surgical resection, radiation therapy and standardized chemotherapy with Temozolimide (TMZ) [21]. The prognosis of GBM patients remains very poor and it's urgent to find new treatments for a better outcome [22]. Immuno- therapy is one of the most promising approaches for cancer treatments [23], which has already been applied to Hodgkin lymphoma [24]. Great efforts have been made to utilize vaccines targeting GBM antigens with little effects [25], suggesting that we need to better understand the biology characteristics of glioma cells to find a more precise target.

It has been reported that TRIM family are important regulators of carcinogenesis as they take part in a lot of cells biological behaviors [10]. Within the big family of

A

HA

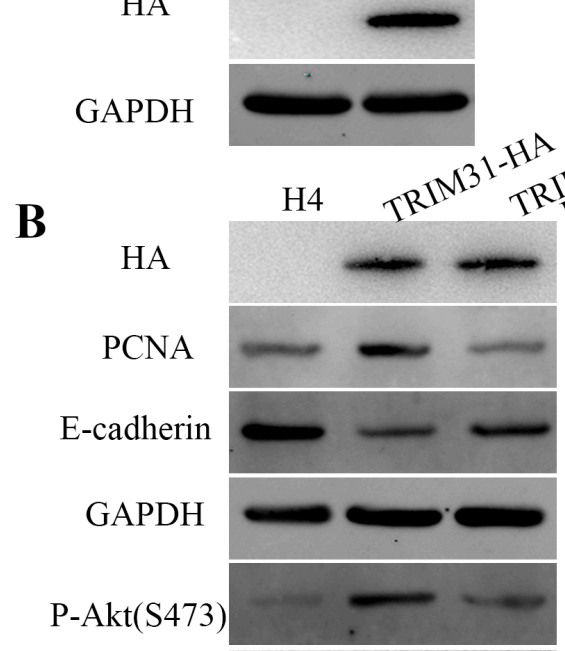

H4 TRIM31-HA

Akt

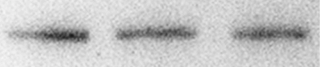

C

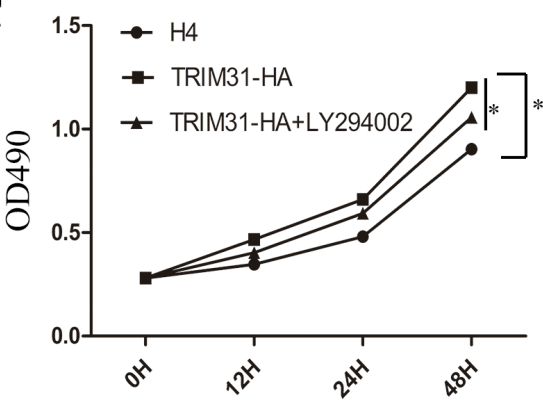

D

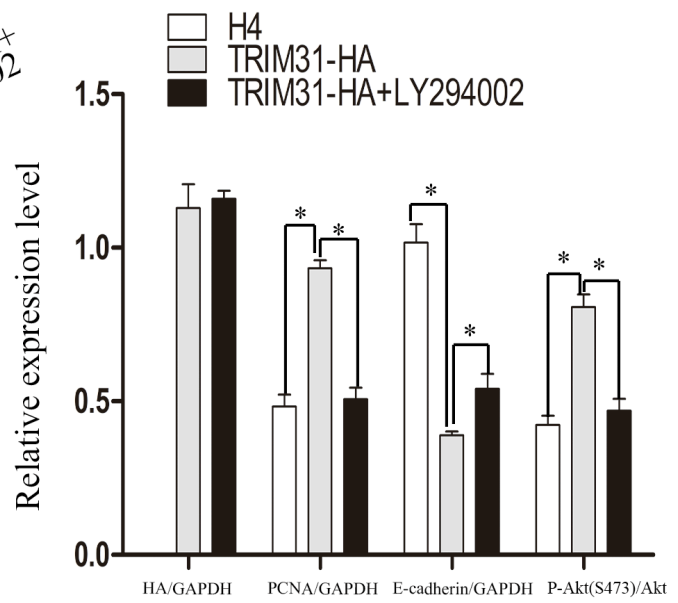

$\mathbf{E}$

$\mathrm{H} 4$

TRIM31--HA

TRIM31-HA+LY294002

Oh
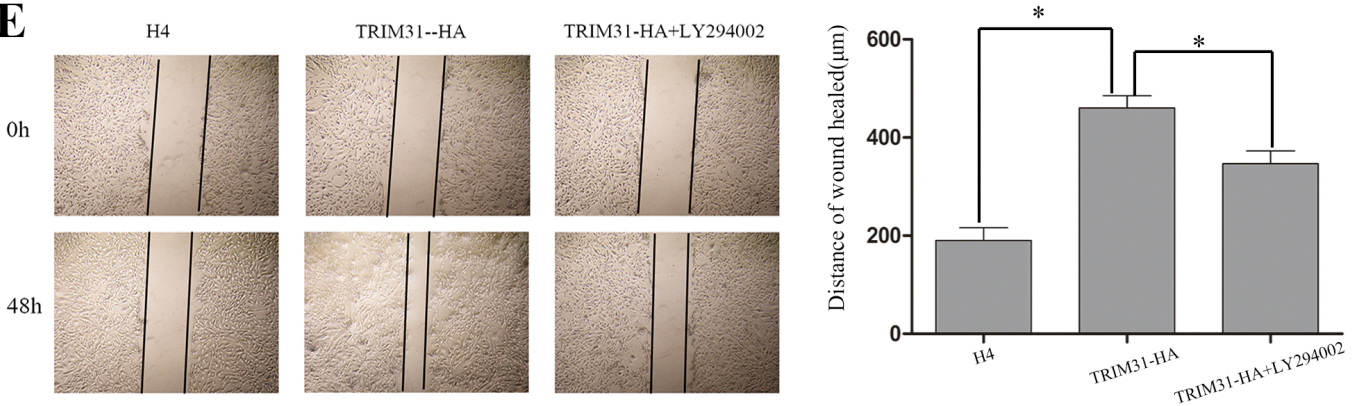

Figure 5. Effects of TRIM31 overexpression and Akt antagonist LY294002 on proliferation, invasion and migration of glioma cells. A) Western blot results of $\mathrm{H} 4$ cells overexpressed with HA labeled TRIM31. B) Western blot results of different procession of H4 cells. Bar charts were TRIM31, PCNA, E-cadherin compared to GAPDH and P-Akt(S473) compared to Akt. ${ }^{\star} \mathbf{p}<0.05$. C) CCK-8 analysis of different procession of $\mathrm{H} 4$ cells. ${ }^{\star} \mathrm{p}<0.05$. D) Transwell invasion assay of different procession of $\mathrm{H} 4$ cells. Bar charts were quantization results of transwell invasion assay. ${ }^{\star} \mathrm{p}<0.05$. E) Wound-healing assay of different procession of $\mathrm{H} 4$ cells. Bar charts were quantization results of wound-healing assay. ${ }^{*} \mathrm{p}<0.05$. 
TRIM proteins, some have been well characterized in tumor progresses [26]. TRIM24, also known as transcriptional intermediary factor $1 \alpha(\mathrm{TIF} \alpha)$, is overexpressed in head and neck squamous cell carcinoma [27] and human cervical cancer [28], promoting their progresses. TRIM27, also named ret finger protein (RFP), can also act as an oncogene and promotes the developments of colorectal cancer [29] and ovarian cancer [30]. TRIM proteins are involved in some mechanisms needed for the maintenance of cellular internal and external environment. However, when their genes were altered, TRIM proteins acquire oncogenic potential [9,31]. Furthermore, several TRIM proteins have a double role in tumor occurrence and development via regulating p53 [32]. Thus, further researches of TRIM proteins' mechanism and involvement in tumorigenesis may provide effective targets for cancer therapies. In this study, we elucidated that TRIM31, as an oncogene, was overexpressed in high-grade gliomas and was a prognostic factor for patients with gliomas.

Vast evidences have verified that Akt signaling pathway plays an important part in glioma occurrence, development, metastasis and aggressiveness [33]. Akt activation is involved in a series of phosphorylation events, mainly through phosphorylation of Akt-Ser473 and Akt-Thr308 [34]. In our study, silence of TRIM31 caused the downregulation of P-Akt(S473), with no effect on P-Akt(T308) (Figure 3C). Moreover, upregulation of TRIM31 promoted the expression of P-Akt(S473) (Figure 5B). We conjectured that TRIM31 modulated the activation of Akt. As knockdown of TRIM31 downregulated the expression of PCNA and E-cadherin (Figure 3B), we speculated that TRIM31 may regulate the proliferation, migration and invasion through Akt signaling pathway. By cell biology experiments, it could be seen that the use of Akt agonist IGF-1 could reverse the inhibition of cell proliferation, invasion and migration induced by shTRIM31 transfection (Figures 4B-D). Conversely, the use of Akt antagonist LY294002 could alleviate the promotion of cell proliferation, invasion and migration induced by TRIM31 overexpression (Figures $5 \mathrm{C}-\mathrm{E}$ ). It is reported that knockdown of TRIM31 suppresses proliferation and invasion of gallbladder cancer cells through the PI3K/Akt signaling pathway [13]. Our experiment results further represented evidences of the relationship between TRIM31 and Akt signaling pathway in glioma cells.

However, our experimental results still need further verification. For example, the sample size needs to be expanded. We will follow up more glioma patients in the future. In addition, our results are based on cell experiments in vitro. The effects of TRIM31 on proliferation, invasion and migration of glioma cells in vivo need to be further interpreted. It has been reported that the dysfunction of inflammatory microenvironment and immune system is closely related to the development of glioma [35], which may influence the effects of TRIM31 on glioma cells. Therefore, we will conduct animal experiments. For in vivo study, sh-NC and sh-TRIM31 U87 stable cell lines will be constructed for inoculation in athymic nude mice through subcutaneous xenograft assay to determine TRIM31-knockdown effects on glioma progression.

This study presented clinical and experimental evidences that TRIM31 contributed to glioma progression. Besides, effects of TRIM31 on proliferation, invasion and migration of glioma cells were associated with activation of Akt signaling pathway. It's proposed that TRIM31 might be a promising molecular target of glioma.

Acknowledgements: This study was supported by grants from the National Natural Science Foundation of China (NO.81572491).

\section{References}

[1] ZHANG S, ZHANG H, YU L. HMGA2 promotes glioma invasion and poor prognosis via a long-range chromatin interaction. Cancer Med 2018; 7: 3226-3239. https://doi. org/10.1002/cam4.1534

[2] BERNTSSON SG, MERRELL RT, AMIRIAN ES, ARMSTRONG GN, LACHANCE D et al. Glioma-related seizures in relation to histopathological subtypes: a report from the glioma international case-control study. J Neurol 2018; 265: 1432-1442. https://doi.org/10.1007/s00415-018-8857-0

[3] ZHANG Y, WANG L. [Up-regulation of GPR37 promotes the proliferation of human glioma U251 cells]. Xi Bao Yu Fen Zi Mian Yi Xue Za Zhi 2018; 34: 341-345.

[4] LIU F, XU K, YANG H, LI Y, LIU J et al. A novel approach to glioma therapy using an oncolytic adenovirus with two specific promoters. Oncol Lett 2018; 15: 3362-3368. https:// doi.org/10.3892/ol.2017.7684

[5] GUO RM, ZHAO CB, LI P, ZHANG L, ZANG SH et al. Overexpression of CLEC18B Associates With the Proliferation, Migration, and Prognosis of Glioblastoma. ASN Neuro 2018; 10. https://doi.org/10.1177/1759091418781949

[6] WESSELING P, CAPPER D. WHO 2016 Classification of Gliomas. Neuropathol Appl Neurobiol 2018; 44: 139-150. https://doi.org/10.1111/nan.12432

[7] GU G, LI W, ZHANG J, WANG H, TAN T et al. MicroRNA-384 inhibits proliferation migration and invasion of glioma by targeting at CDC42. Onco Targets Ther 2018; 11: 4075-4085. https://doi.org/10.2147/OTT.S166747

[8] HALL AW, BATTENHOUSE AM, SHIVRAM H, MORRIS AR, COWPERTHWAITE MC et al. Bivalent Chromatin Domains in Glioblastoma Reveal a Subtype-Specific Signature of Glioma Stem Cells. Cancer Res 2018; 78: 2463-2474. https://doi.org/10.1158/0008-5472.CAN-17-1724

[9] OZATO K, SHIN DM, CHANG TH, MORSE HC 3RD. TRIM family proteins and their emerging roles in innate immunity. Nat Rev Immunol 2008; 8: 849-860. https://doi. org/10.1038/nri2413.

[10] NISOLE S, STOYE JP, SAIB A. TRIM family proteins: retroviral restriction and antiviral defence. Nat Rev Microbiol 2005; 3: 799-808. https://doi.org/10.1038/nrmicro1248 
[11] DOKMANOVIC M, CHANG BD, FANG J, RONINSON IB. Retinoid-induced growth arrest of breast carcinoma cells involves co-activation of multiple growth-inhibitory genes. Cancer Biol Ther 2002; 1: 24-27.

[12] Wang H, Yao L, Gong Y, Zhang B. TRIM31 regulates chronic inflammation via NF- $\mathrm{\kappa B}$ signal pathway to promote invasion and metastasis in colorectal cancer. Am J Transl Res 2018: 1247-1259.

[13] LI H, ZHANG Y, HAI J, WANG J, ZHAO B et al. Knockdown of TRIM31 suppresses proliferation and invasion of gallbladder cancer cells by down-regulating MMP2/9 through the PI3K/Akt signaling pathway. Biomed Pharmacother 2018; 103: 1272-1278. https://doi.org/10.1016/j.biopha.2018.04.120

[14] GUO P, MA X, ZHAO W, HUAI W, LI T et al. TRIM31 is upregulated in hepatocellular carcinoma and promotes disease progression by inducing ubiquitination of TSC1-TSC2 complex. Oncogene 2017; 37: 478-488. https://doi.org/10.1038/ onc.2017.349

[15] LI H, ZHANG Y, ZHANG Y, BAI X, PENG Y et al. TRIM31 is downregulated in non-small cell lung cancer and serves as a potential tumor suppressor. Tumour Biol 2014; 35: 57475752. https://doi.org/10.1007/s13277-014-1763-x

[16] YAMADA NO, WENDUERMA, MATSUDA S, SENDA T. Validation and application of a novel APC antibody in western blotting, immunoprecipitation, and immunohistochemistry. Med Mol Morphol 2018; 51: 227-236. https://doi. org/10.1007/s00795-018-0196-9

[17] MUSUMECI G, CASTROGIOVANNI P, MAZZONE V, SZYCHLINSKA MA, CASTORINA S et al. Histochemistry as a unique approach for investigating normal and osteoarthritic cartilage. Eur J Histochem 2014; 58: 2371. https://doi. org/10.4081/ejh.2014.2371

[18] NIELSEN LAG, BANGSO JA, LINDAHL KH, DAHLROT RH, HJELMBORG JVB et al. Evaluation of the proliferation marker Ki-67 in gliomas: Interobserver variability and digital quantification. Diagnostic Pathology 2018; 13: 38. https:// doi.org/10.1186/s13000-018-0711-2

[19] SAWA M, YABUKI A, SETOGUCHI A, YAMATO O. Development and application of multiple immunofluorescence staining for diagnostic cytology of canine and feline lymphoma. Vet Clin Pathol. 2015; 44: 580-585. https://doi. org/10.1111/vcp.12300

[20] QIU X, HE X, HUANG Q, LIU X, SUN G et al. Overexpression of CCT8 and its significance for tumor cell proliferation, migration and invasion in glioma. Pathol Res Pract 2015; 211: 717-725. https://doi.org/10.1016/j.prp.2015.04.012

[21] GUAN Y, HE Y, LV S, HOU X, LI L et al. Overexpression of HOXC10 promotes glioblastoma cell progression to a poor prognosis via the PI3K/AKT signalling pathway. J Drug Target 2019; 27: 60-66. https://doi.org/10.1080/106118 6X.2018.1473408

[22] DONATE-MACIAN P, GOMEZ A, DEGANO IR, PERALVAREZ-MARIN A. A TRPV2 interactome-based signature for prognosis in glioblastoma patients. Oncotarget 2018; 9: 18400-18409. https://doi.org/10.18632/oncotarget.24843
[23] DU X, FEI T, LIU M, SU J, ZHANG Y et al. A reappraisal of CTLA-4 checkpoint blockade in cancer immunotherapy. Cell Research 2018; 28: 416-432. https://doi.org/10.1038/ s41422-018-0011-0

[24] MICHOT JM, LAZAROVICI J, GHEZ D, DANU A, FER$\mathrm{ME} \mathrm{C}$ et al. Challenges and perspectives in the immunotherapy of Hodgkin lymphoma. Eur J Cancer 2017; 85: 67-77. https://doi.org/10.1016/j.ejca.2017.08.014.

[25] PLATTEN M. Immunotherapy of Gliomas, pp 657-664. In: L Zitvogel, G Kroemer (Eds.). Oncoimmunology. A Practical Guide for Cancer Immunotherapy. Springer International Publishing 2018, p 724. ISBN 978-3-319-62430-3.

[26] HATAKEYAMA S. TRIM proteins and cancer. Nat Rev Cancer 2011; 770: 792-804. https://doi.org/10.1038/nrc3139

[27] CUI Z, CAO W, LI J, SONG X, MAO L et al. TRIM24 overexpression is common in locally advanced head and neck squamous cell carcinoma and correlates with aggressive malignant phenotypes. PLoS One 2013; 8: e63887. . https://doi. org/10.1371/journal.pone.0063887

[28] LIN L, ZHAO W, SUN B, WANG X, LIU Q. Overexpression of TRIM24 is correlated with the progression of human cervical cancer. Am J Transl Res 2017; 9: 620-628.

[29] ZHANG Y, FENG Y, JI D, WANG Q, QIAN W et al. TRIM27 functions as an oncogene by activating epithelial-mesenchymal transition and p-AKT in colorectal cancer. Int J Oncol 2018; 53: 620-632. https://doi.org/10.3892/ijo.2018.4408

[30] HORIO M, KATO T, MII S, ENOMOTO A, ASAI M et al. Expression of RET finger protein predicts chemoresistance in epithelial ovarian cancer. Cancer Med 2012; 1: 218-229. https://doi.org/10.1002/cam4.32

[31] CAMBIAGHI V, GIULIANI V, LOMBARDI S, MARINELLI C, TOFFALORIO F et al. TRIM Proteins in Cancer. Adv Exp Med Biol 2012; 770: 77-91.

[32] KUNG CP, KHAKU S, JENNIS M, ZHOU Y, MURPHY ME. Identification of TRIML2, a Novel p53 Target, that Enhances p53 SUMOylation and Regulates the Transactivation of Proapoptotic Genes. Mol Cancer Res 2015; 13: 250-262. https://doi.org/10.1158/1541-7786.MCR-14-0385

[33] WANG R, DENG D, SHAO N, XU Y, XUE L et al. Evodiamine activates cellular apoptosis through suppressing PI3K/ AKT and activating MAPK in glioma. Onco Target Ther 2018; 11: 1183-1192. https://doi.org/10.2147/OTT.S155275

[34] XUE L, HUANG J, ZHANG T, WANG X, FU J et al. PTEN inhibition enhances angiogenesis in an in vitro model of ischemic injury by promoting Akt phosphorylation and subsequent hypoxia inducible factor-1 $\alpha$ upregulation. Metab Brain Dis 2018; 33: 1679-1688. https://doi.org/10.1007/ s11011-018-0276-5

[35] XIE T, MA JW, LIU B, DONG J, HUANG Q. Experimental study of glioma stem cell-mediated immune tolerance in tumor microenvironment. Zhonghua Zhong Liu Za Zhi 2017; 39: 808-813. https://doi.org/10.3760/cma.j.is sn.0253-3766.2017.11.002 